\title{
A Hybrid Finger Identification Pattern Using Polarized Depth-weighted Binary Direction Coding
}

\author{
Wenming Yang ${ }^{\mathrm{a}, *}$, Wenyang Ji ${ }^{\mathrm{a}}$, Jing-Hao Xue ${ }^{\mathrm{b}}$, Yong Ren ${ }^{\mathrm{a}}$, Qingmin Liao ${ }^{\mathrm{b}}$ \\ ${ }^{a}$ Shenzhen Key Lab of Information SciETech / Shenzhen Engineering Lab of ISEDCP, China; \\ Department of Electronic Engineering / Graduate School at Shenzhen, Tsinghua University, China \\ ${ }^{b}$ Department of Statistical Science, University College London, UK
}

\begin{abstract}
Finger identification is increasingly popular in recent years. In this paper, we propose a new finger identification system to acquire a hybrid pattern of dorsal finger vein and texture in a single image by using only one camera. It effectively reduces the cost and volume of the imaging device to acquire multi-modal patterns. The hybrid pattern of dorsal finger vein and texture is both storage-saving and calculation-saving. As there was no existing method specially developed for this kind of pattern, we propose a new feature extraction method called "Polarized depth-Weighted Binary Direction Coding" (PWBDC). We also establish a new database of such hybrid images of 210 finger samples. Experimental results demonstrate that the proposed system and feature are not only storage and calculation saving, but more importantly effective for identification. The proposed PWBDC method performs well on both the newly established database of hybrid images and a popular public database of traditional finger vein images, superior to many established and state-of-the-art methods.
\end{abstract}

Keywords: Finger identification, Finger vein, Finger dorsal texture, Direction coding

\section{Introduction}

Human identification using biometric identifiers is increasingly popular in recent years. Among the widely-used biometric identifiers, finger vein is an important one due to its acquisition convenience and relatively high recognition accuracy.

On the process of finger vein identification, several comprehensive analyses have been reported. For example, [2] summarized the finger vein authentication technology and made an outlook for its future; [15] studied approaches to improving the quality of finger vein images affected by light variation and noise, as well as adaptive thresholding and template matching; and [21] presented the Sobel detector, enhancement filter and binarization process.

In the process of finger vein identification, there are often three steps. The first step is preprocessing, including region of interest (ROI) extraction, image enhancement and normalization [27, 33, 31]. The second step is feature extraction, in which finger vein features are extracted. The third step is matching $[25,24,14]$, in which the extracted features from probe finger vein images are compared to those from the samples in a database, and the class of the sample with the highest matching score is chosen for the probe images.

Among these three steps, finger vein feature extraction is the most important step, on which great efforts have been continuously made. For example, a modified Gaussian high-pass filter was used in [7]; a series of Gabor filters were applied to

\footnotetext{
${ }^{*}$ Corresponding author.

Email address: yangelwm@163.com (Wenming Yang)
}

infrared images to determine the most representative direction in [4] and [28]; a descriptor of local ternary cooccurrence patterns was proposed by [20] to represent finger veins in a local region; a new texture descriptor called local line binary pattern was utilized by [16] to offer better performance than the local binary pattern; and some soft biometric traits in finger veins were used by [30]. Some other methods focus on the locations of veins in the image. A modified separable Mumford Shah model was proposed in [23] to segment finger veins from infrared images. The local maximum curvature method was proposed in [12] to investigate the gray-value variation in the cross section of veins, while the repeated line tracking was introduced in [11] to trace veins through their orientations. Another representative method was proposed in [33] to use the cross section to determine locations of veins and code directions in the vein area. A deep representation-based feature was explored in [17], achieving good performance compared to traditional handcrafted features.

However, all the methods aforementioned use unimodal features only. Finger vein patterns are usually indispensable in identification, because they have the advantage of being much more difficult to fake than surface features such as finger dorsal textures. However, neither the finger vein pattern nor the finger dorsal texture is sufficient for personal identification. For example, some girls' finger veins are so tenuous, as Fig. 1.a shows, that it is hard to observe the vein pattern clearly using the nearinfrared images acquired from the backside of the finger. Therefore, finger dorsal textures, as shown in Fig. 1.b, should also be considered, in order to attain more comprehensive information and better performance of finger identification. 


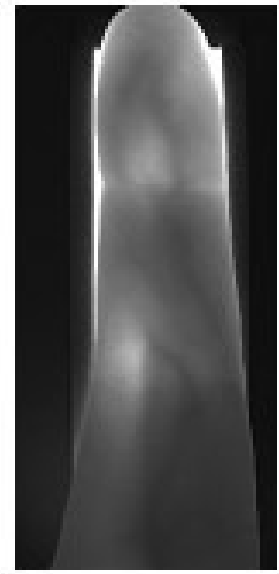

a

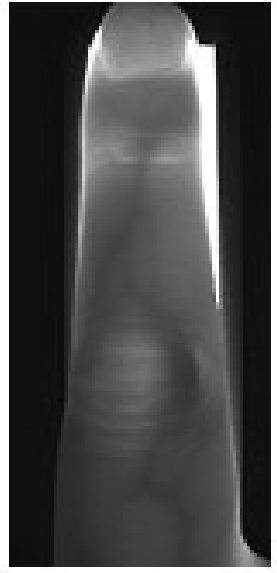

b
Figure 1: (a) is the traditional finger vein pattern of a girl; (b) is our proposed hybrid pattern with information of both dorsal finger texture and dorsal finger vein embedded in a single image.

Indeed, besides the feature-level fusion of finger print and finger vein as investigated in [29] and [22], Yang and Huang [31] also fused the finger dorsal textures and finger veins at the feature level and achieved better results than using the finger veins alone. However, there are two limitations of such a feature fusion. Firstly, the finger veins are acquired by using infrared light (Fig. 2.a) but the finger dorsal textures are obtained by using natural light (Fig. 2.b), and hence they cannot be stored in a single image, which is storage consuming. Secondly, these two types of features have to be extracted separately from two different images and aligned before fusion, which may lead to sub-optimal performance of finger identification.

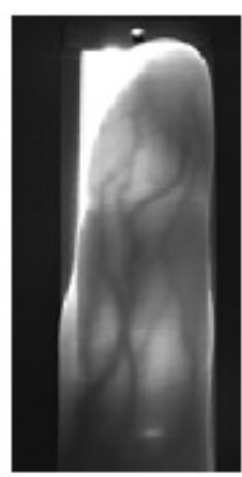

a

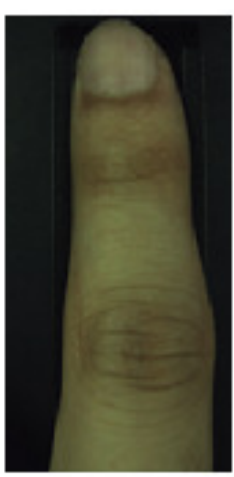

b

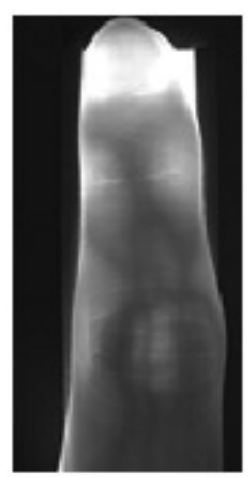

C
Figure 2: (a) and (b) show the traditional finger vein pattern (under infrared light) and finger dorsal texture pattern (under natural light); (c) shows the proposed hybrid pattern (both under infrared light).

Therefore, in this paper we propose a new system to acquire dorsal finger vein and texture into a single image, as illustrated by Fig. 2.c, using infrared light because of its strong penetration ability. An imaging device is designed as Fig. 3.a shows, which can capture the hybrid pattern of dorsal finger vein and texture in a single image simultaneously. Compared with traditional multi-modal schemes, the new system effectively reduces the cost and volume of imaging device. Because we deal with the hybrid pattern in only one image, the storage space and the calculation amount can also be saved. Considering that there was no existing method specially developed for the hybrid pattern of dorsal finger vein and texture, We present a new method to extract the hybrid pattern from the image for finger identification. We shall show that the proposed system and feature are not only storage-saving, but more importantly identification-effective.

The key contributions of our work are threefold:

1. We develop a new system to capture the hybrid pattern of dorsal finger vein and texture with only a single imaging device. It is cost-saving and volume-saving compared with traditional capturing devices.

2. For the hybrid pattern of dorsal finger vein and texture, we develop a new hybrid finger feature and its extraction method, which is termed Polarized depth-Weighted Binary Direction Coding (PWBDC); this hybrid finger feature can be effective for biometric identification.

3. We establish a database of 210 samples, extending from a database of 126 samples [32], which will be published in the future.

The rest of the paper is organized as follows. Section 2 describes briefly our new image acquisition device. Section 3 reviews four classical feature extraction methods used in finger vein recognition, LBP [16], GCC [4], LMC [12] and LDC [33], as our evaluation baselines. Section 4 details our proposed PWBDC method, including three innovative components: polarized direction extraction, extended normalized angular binary coding, and self-adaptive depth-dependent weighting. Section 5 presents our experiments and results. Conclusions and some future work are discussed in section 6 .

\section{Image Acquisition Device}

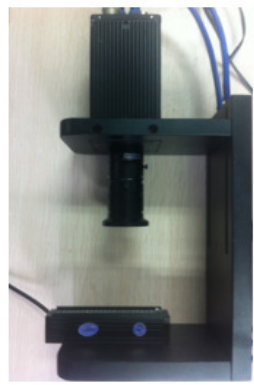

a

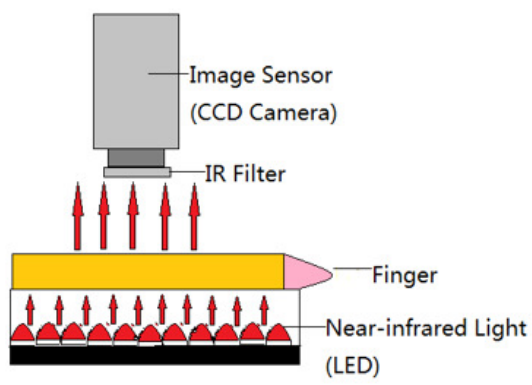

b
Figure 3: (a) is our self-designed image capture device; (b) is its diagram and physical principle.

There were many unimodal vein image acquisition systems, for example, for hand [26] and for finger [9]. However, as far as we are concerned, there is no device to capture a single image blending both finger vein and finger dorsal texture, such as 
Fig. 1.b and Fig. 2.c. Therefore, we design and develop a new image acquisition device, as shown in Fig. 3.

In Fig. 3.a, a black JAI camera is placed above, and a rectangular near-infrared illuminant is positioned below. The physical principle (illustrated in Fig. 3.b) is explained as follows. Firstly, near-infrared light penetrates a finger, and then those passing through blood vessels is absorbed by the hemoglobin in red blood cells to some extent, so the vein area will look darker than other parts and thus can be captured.

Compared with traditional devices, the new device does not bring additional costs, because the CCD camera of the new designed device can almost be replaced by a traditional one. The difference between traditional devices and the newly designed device mainly exists in two aspects. Traditional devices capture the finger inner vein, while the proposed device captures the finger dorsal vein, which is nearer to the finger skin surface and more remarkable than the finger inner vein. Along with the finger dorsal vein, the newly designed device also simultaneously captures the finger dorsal skin texture. As Fig. 2.c shows, the proposed device can capture both the finger dorsal vein and the finger dorsal skin texture, and store the information of both modalities into a single image simultaneously, which saves storage and brings richer feature. In our case, one CCD camera is sufficient with no additional costs incurred. Since finger dorsal texture is sunken, the part of finger corresponding to this texture is thinner than other parts. Thus, infrared light more easily penetrates this part of finger and arrives at camera when imaging. In addition, more scattering and refraction light will gather in the tinny sunken space, which makes the finger dorsal texture look bright and visible. However, the finger dorsal texture overlapping with veins seems lost, since veins will absorb more infrared light and prevent the light from reaching camera.

\section{Classical Feature Extraction and Matching Methods}

Feature extraction and matching are two crucial components of the process of finger identification. Here we first review four classical methods of finger-vein feature extraction, LBP [16], GCC [4], LMC [12], and LDC [33], as well as their matching methods, which are widely applied in finger identification; they will act as the baselines in the experiments presented in this paper.

\subsection{Local Binary Patterns (LBP)}

The local binary pattern (LBP) method [16] is widely used as a baseline feature-extraction scheme, to validate the reliability of a database and finger-identification algorithms. Its core concept is to compare the values of neighboring pixels with a given central pixel:

$$
L B P_{P}=\sum_{p=0}^{P-1} s\left(g_{p}-g_{c}\right) 2^{p}, \quad s(x)=\left\{\begin{array}{l}
1, x \geq 0 \\
0, x<0,
\end{array}\right.
$$

where $g_{c}$ is the value of the central pixel; $g_{p}$ is the value of neighboring pixels; and $P$ is the total number of neighboring pixels.

A pixel-by-pixel matching method is applied to the final coded images.

\subsection{Gabor Competitive Coding (GCC)}

The Gabor competitive coding (GCC) method was proposed in [4]. It is a widely-used method to exploit the local direction information. In biometric pattern recognition, the Gabor filters have been widely used in feature extraction because of its superior performance in containing the information of magnitude, phase and orientation simultaneously in a single formula. The Gabor filter can be defined as follows:

$$
G\left(x_{\theta}, y_{\theta}, \gamma, \sigma, f_{0}\right)=\frac{\gamma}{2 \pi \sigma^{2}} e^{-\frac{1}{2}\left(\frac{x_{\theta}^{2}+\gamma^{2} y_{\theta}^{2}}{\sigma^{2}}\right)} e^{j 2 \pi f_{0} x_{\theta}},
$$

where $\sigma$ is the standard deviation of the Gaussian function; $\gamma$ is the adjustment factor for the Gaussian envelope in the y direction, which is in fact the standard deviation ratio between directions $\mathrm{x}$ and $\mathrm{y} ; f_{0}$ is the center frequency of the Gabor filter; $\theta$ is the orientation parameter; and $\left(x_{\theta}, y_{\theta}\right)$ is the rotated version of the coordinate $(x, y)$.

For our experiments in section 5, six orientations are selected, which is consistent with the conclusion made in [8] that most neural cells are very sensitive to specific orientations with bandwidth of $\pi / 6$. The Gabor feature map is then transformed into 3-bit binary code and finally a bitwise matching method is applied to the two coded feature maps.

\subsection{Local Maximum Curvature (LMC)}

The local maximum curvature (LMC) method to extract the vein feature was originally proposed in [12]. It can extract the center lines of finger veins without being much affected by the vein width and brightness variation, and thus can enhance the robustness and matching precision. The algorithm contains three steps: extraction of center positions by using local maximum curvature, connection of center positions, and matching. The curvature is calculated as

$$
\kappa(z)=\frac{d^{2} P_{f}(z) / d z^{2}}{\left\{1+\left(d P_{f}(z) / d z\right)^{2}\right\}^{\frac{3}{2}}},
$$

where $P_{f}(z)$ is a cross-sectional profile with selected direction in the original image, and $z$ is a point on this cross section.

In the matching step, a score indicating the probability of a center position on the vein is calculated as

$$
S_{c r}\left(z_{i}^{\prime}\right)=\kappa\left(z_{i}^{\prime}\right) \times W_{r}\left(z_{i}^{\prime}\right),
$$

where $\kappa\left(z_{i}^{\prime}\right)$ is the maximum curvature calculated in Eq.(3) and $W_{r}\left(z_{i}^{\prime}\right)$ is the width of the point $z_{i}^{\prime}$ whose curvature is positive. In our experiments, we find that the local curvature of points is about $-10 \sim 5$, and the width of the vein area of positive curvature is about $8 \sim 12$ pixels.

\subsection{Local Direction Coding (LDC)}

Another method often used in finger vein feature extraction is the local direction coding (LDC) method proposed in [33]. The difference in brightness is often used in the vein feature extraction. The LDC method selects the deepest direction into 
the feature map. The formula calculating the depth of a selected position is

$$
\begin{aligned}
G_{\theta}(i, j) & =I(i-2 r \sin \theta, j-2 r \cos \theta) \\
& +I(i-r \sin \theta, j-r \cos \theta) \\
& +I(i+r \sin \theta, j+r \cos \theta) \\
& +I(i+2 r \sin \theta, j+2 r \cos \theta) \\
& -4 I(i, j)
\end{aligned}
$$

where $\theta$ is the vein direction, and $4 r$ is the estimated vein width. In our experiments, the vein width is about $8 \sim 12$ pixels, so the average vein width is 10 pixels and $r$ is set to 2.5 . Six directions are selected in the final extracted feature map.

In the matching step, a bitwise matching method is applied on the binary coded feature maps, and the one with the highest score is selected as the matched finger pattern.

\section{Polarized depth-Weighted Binary Direction Coding (PWBDC)}

Because the finger veins and finger dorsal textures are captured in a single image, we need to develop a new direction coding scheme for extracting hybrid finger features to represent a hybrid finger-identification pattern. We call our proposed feature extraction method "polarized depth-weighted binary direction coding (PWBDC)" approach, which includes three innovative major components: polarized direction extraction, extended normalized angular binary coding, and self-adaptive depthdependent weighting. The first component, polarzied direction extraction, is to construct feature maps for the two deepest directions over an image. The second component, extended normalized angular binary coding, is to provide an appropriate coding of each direction feature map. The third component, selfadaptive depth-dependent weighting, is to adaptively weight the contributions of the two deepest directions in the matching.

The diagram of PWBDC is illustrated in Fig. 5. The portion in the red dotted wireframe shows our proposed method applied on the probe image $P$, and the processing steps for the image $T$ in database are similar illustrated in the symmetric lower part of Fig. 5. In our experiments, the binary coded direction maps $B_{k_{1}}^{\prime}$, $B_{k_{2}}^{\prime}$ and the corresponding depth maps $G_{k_{1}}^{\prime}$ and $G_{k_{2}}^{\prime}$ of images $T$ in the database are calculated in advance. When a probe image $P$ comes, its binary coded direction maps $B_{k_{1}}$ and $B_{k_{2}}$, as well as the corresponding depth maps $G_{k_{1}}$ and $G_{k_{2}}$, are figured out, then a self-adaptive depth-weighted bitwise matching is performed with every $T$ in the database, and finally the one with the highest matching score $S(P, T)$ is chosen as the matched sample.

\subsection{Polarized Direction Extraction}

In the LDC method [33], only one direction with the deepest depth is selected as the feature, which unfortunately is not able to extract sufficient information from our images, where both finger vein information and finger dorsal texture information are embedded in a single image. Therefore, we propose to simply select the first two deepest directions.

Take Fig. 4.a as an illustrative example, in which the yellow lines in the two regions represent the real dorsal finger vein and texture direction, and eight candidate directions are marked as

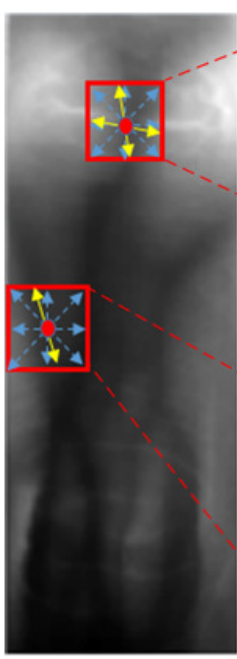

a

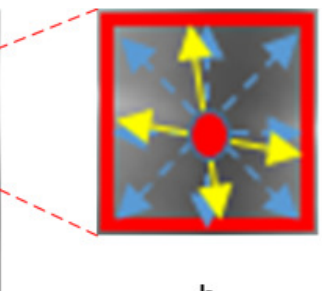

b

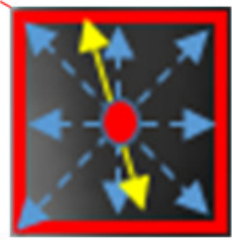

d
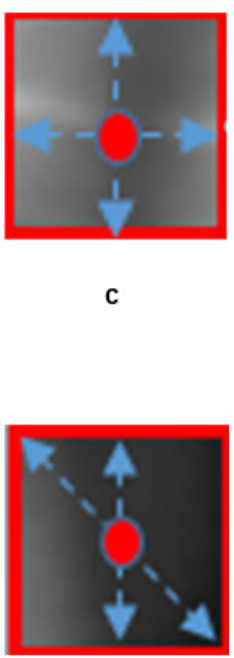

e
Figure 4: (a) is the image to be processed; (b) and (d) are two typical regions; and (c) and (e) are the corresponding coded directions.

dashed blue lines. The benefit from using the two deepest directions instead of one can be illustrated in two cases. As Fig. 4.b shows, in the case of junction points of vertical finger vein and horizontal finger dorsal texture, the two deepest candidate directions are the closest to the vertical finger vein and horizontal finger dorsal texture. In the other case where there is only one significant orientation, as Fig. 4.d shows, the two deepest candidate directions are exactly its two neighboring directions. The finally extracted directions are shown in Fig. 4.c and Fig. 4.e, respectively; in both cases, the two selected directions represent the situations better than using a single candidate direction. As a result, through adding the information of a second depth, both the "vein valley" and the "finger dorsal texture peak" can be taken into account. Also, for the points only in the "valley" or on the "peak", more information can be recorded for the next matching step.

The brightness of vein position is usually darker than other positions, so $G_{k}(i, j)$ in vein positions is usually larger than other positions. Technically, the depths of the eight different directions are first calculated as

$$
\begin{aligned}
G_{k}(i, j) & =\operatorname{abs}\left(I\left(i-2 r \sin \theta_{k}, j-2 r \cos \theta_{k}\right)\right. \\
& +I\left(i-r \sin \theta_{k}, j-r \cos \theta_{k}\right) \\
& +I\left(i+r \sin \theta_{k}, j+r \cos \theta_{k}\right) \\
& +I\left(i+2 r \sin \theta_{k}, j+2 r \cos \theta_{k}\right) \\
& -4 I(i, j)), \\
\theta_{k} & =(k-1) \times 22.5^{\circ} \text { for } k=1, \ldots, 8,
\end{aligned}
$$

where $G_{k}$ represents the depth of the $k$ th direction. Then the deepest depth $G_{k_{1}}$ and the second deepest depth $G_{k_{2}}$ are selected and recorded as

$$
\left(G_{k_{1}}, G_{k_{2}}\right)=\max _{k_{1}, k_{2}=1, \ldots, 8}\left\{G_{1}, \ldots, G_{8}\right\} .
$$

Finally, the two recorded directions $k_{1}$ and $k_{2}$ of all related pixels constitute two direction feature maps, and the corresponding two deepest depths $G_{k_{1}}$ and $G_{k_{2}}$ will be further used to calculate 


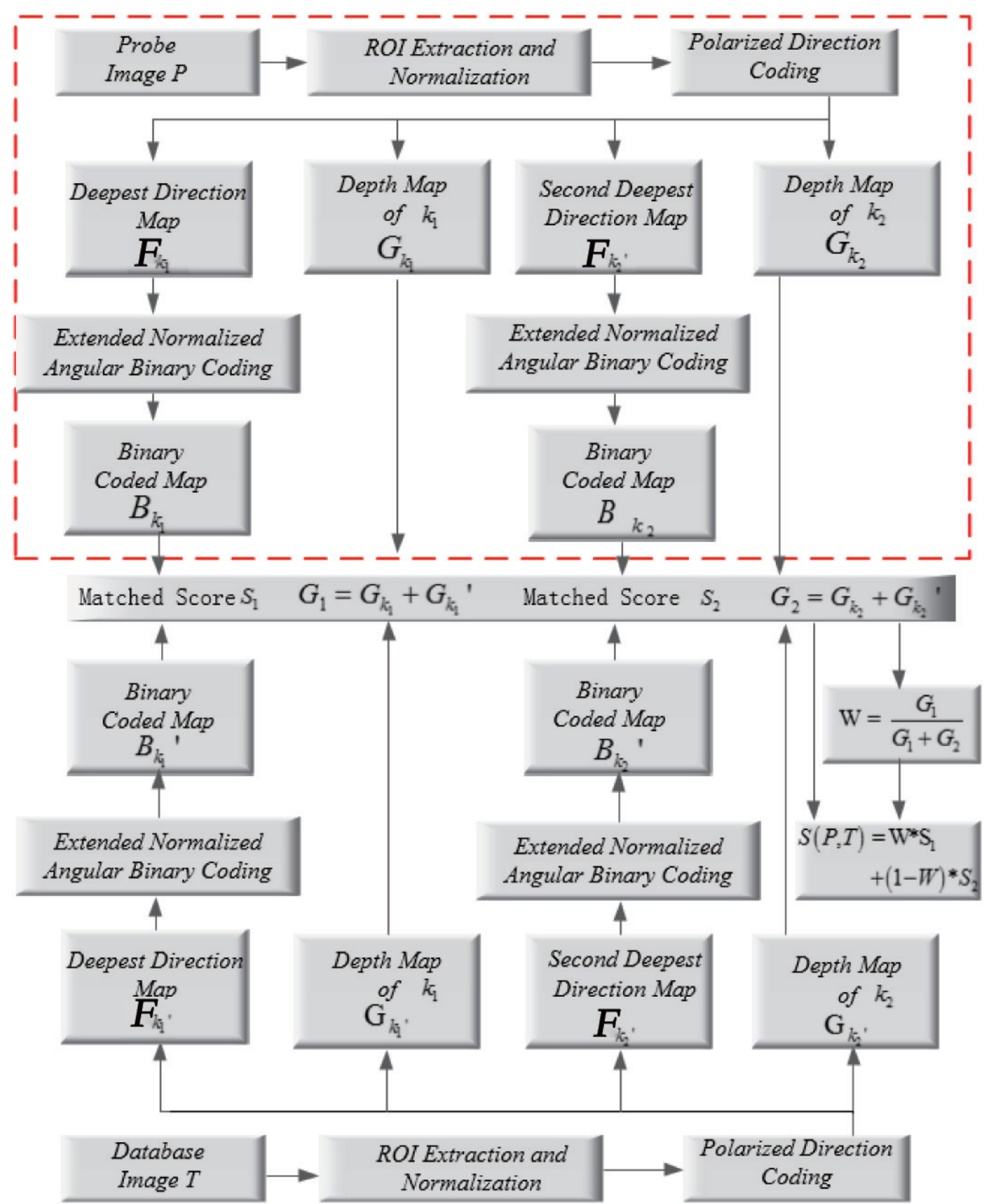

Figure 5: The diagram of the major steps of our proposed PWBDC scheme, where $W$ is the calculated weight, and $S$ is the final matching score between a probe image $P$ and a database image $T$. 
Table 1: The extended normalized angular binary coding.

\begin{tabular}{lrrrr}
\hline Direction & bit1 & bit2 & bit3 & bit4 \\
\hline 0 & 0 & 0 & 0 & 0 \\
1 & 0 & 0 & 0 & 1 \\
2 & 0 & 0 & 1 & 1 \\
3 & 0 & 1 & 1 & 1 \\
4 & 1 & 1 & 1 & 1 \\
5 & 1 & 1 & 1 & 0 \\
6 & 1 & 1 & 0 & 0 \\
7 & 1 & 0 & 0 & 0 \\
\hline
\end{tabular}

the depth-based confidence weights for the fusion of matching scores, as we shall show later in section 4.3.

\subsection{Extended Normalized Angular Binary Coding}

After obtaining the two deepest direction feature maps of one probe image $P, F_{k_{1}}$ and $F_{k_{2}}$, and those of the database image $T, F_{k_{1}}^{\prime}$ and $F_{k_{1}}^{\prime}$, we will calculate the matching score between the feature maps. People often first code the feature maps and then calculate the distance between the codes to obtain the matching score. However, for the direction maps that we obtain, the traditional binary coding scheme may not be appropriate.

For instance, for a specific pixel of two feature maps, suppose the directions of a pair of points to match are decimally coded as 5 and 4, and for another pair are 5 and 1. Apparently, the difference between the first paired direction is smaller than that between the second paired directions. However, when the simple binary coding method is used, the direction 5 will be coded as 101, the direction 4 will be coded as 100 , and hence the Hamming distance between them is 1 bit. Meanwhile, the direction 1 will be coded as 001, and hence the Hamming distance between directions 5 and 1 will also be only 1 bit, which is the same as the Hamming distance between directions 5 and 4 .

For such direction maps, the angular binary coding strategy [4] is more appropriate. However, the original angular coding was only for six directions. Hence we propose an extended normalized angular binary coding for eight directions as we need. The coding scheme is designed in Table 1.

This coding method in Table 1 follows the principle that the difference between adjacent orientations is small. Take the previous example again, now the direction 5 will be coded as 1110 , and the direction 4 will be coded as 1111, so the Hamming distance between them is only 1 bit. The direction 1 will be coded as 0001 , so the Hamming distance between directions 5 and 1 will be 4 bits, which is much greater than that of directions 5 and 4 . In this way, the direction information will be used more appropriately in the next step of matching.

Here, for the probe image in Fig. 5, by using the extended normalized angular binary coding method, the deepest direction map $F_{k_{1}}$ and the second deepest direction map $F_{k_{1}}$ are coded as $B_{k_{1}}$ and $B_{k_{2}}$, respectively.

\subsection{Self-adaptive Depth-based Weighting}

Now we have obtained two binary coded polarized direction maps for a probe image $P, B_{k_{1}}$ and $B_{k_{2}}$, and their corresponding depth maps $G_{k_{1}}$ and $G_{k_{2}}$. Similarly for the database image $T$, the coded maps are denoted by $B_{k_{1}}^{\prime}$ and $B_{k_{2}}^{\prime}$, and the corresponding depth maps $G_{k_{1}}^{\prime}$ and $G_{k_{2}}^{\prime}$. To identify the probe finger in $P$, we need a strategy for matching the two binary coded direction maps $B_{k}$ and $B_{k}^{\prime}$, as well as for fusing the matching scores $S_{1}$ and $S_{2}$ of the deepest and the second deepest direction maps.

Firstly, to calculate the matching score between $B_{k}$ and $B_{k}^{\prime}$, we propose

$$
\begin{aligned}
& S_{1}=\frac{2 \phi\left(B_{k_{1}}, B_{k_{1}}^{\prime}\right)}{4\left(N\left(F_{k_{1}}\right)+N\left(F_{k_{1}}^{\prime}\right)\right)}, \\
& S_{2}=\frac{2 \phi\left(B_{k_{2}}, B_{k_{2}}^{\prime}\right)}{4\left(N\left(F_{k_{2}}\right)+N\left(F_{k_{2}}^{\prime}\right)\right)},
\end{aligned}
$$

where

$$
\begin{aligned}
& N(k)=\sum_{i=1}^{m} \sum_{j=1}^{n} g(k(i, j)), \\
& g(x)= \begin{cases}1, & x>0 ; \\
0, & x=0,\end{cases} \\
& \phi\left(B, B^{\prime}\right)=\sum_{i=1}^{m} \sum_{j=1}^{n} \sum_{b=1}^{4} \delta\left(B(i, j, b), B^{\prime}(i, j, b)\right),
\end{aligned}
$$

and

$$
\delta(x, y)= \begin{cases}1, & x \neq 0, y \neq 0, x=y \\ 0, & \text { otherwise }\end{cases}
$$

In Eq.(8) and Eq.(9), $N(k)$ represents the number of nonzero elements of map $k$, and $\phi\left(B, B^{\prime}\right)$ is the matched bit number of nonzero elements in the binary coded maps $B$ and $B^{\prime}$. Because the directions are encoded in 4 bits, 4 appears in the denominator; because $\phi\left(B, B^{\prime}\right)$ is the matched number of two direction maps $B$ and $B^{\prime}, 2$ appears in the numerator to normalize the matching scores $S_{1}$ and $S_{2}$ to range [0, 1].

Secondly, it is reasonable that the deepest direction map and the second deepest direction map do not have the equal significance in feature representation and thus in the calculation of final matching score. Because actually a lot of points are not junctions, and they are neither in the "vein valley" nor on the "dorsal texture peak", the deepest depth may be much larger than the second deepest depth. Therefore, based on the assumption that the directions with a deeper depth have a stronger ability in feature representation, we propose a self-adaptive depthdependent weighting scheme to fuse $S_{1}$ and $S_{2}$ :

$$
S(P, T)=W \times S_{1}+(1-W) \times S_{2},
$$

where

$$
\begin{aligned}
& W=\frac{G_{1}}{G_{1}+G_{2}}, \\
& G_{1}=G_{k_{1}}+G_{k_{1}}^{\prime}, \\
& G_{2}=G_{k_{2}}+G_{k_{2}}^{\prime} .
\end{aligned}
$$


In Eq.(14), $S(P, T)$ denotes the final matching score between probe image $P$ and database image $T ; S_{1}$ and $S_{2}$ are the matching scores of the deepest and second deepest direction maps, respectively; and $W$ is the self-adaptive weight to reflect the relative influence of the deepest and second deepest depths. In Eq.(16) and Eq.(17), $G_{1}$ and $G_{2}$ are the sums of the deepest and second deepest depths $G_{k_{1}}$ and $G_{k_{1}}^{\prime}$, and $G_{k_{2}}$ and $G_{k_{2}}^{\prime}$, respectively. As $G_{1}$ is always larger than $G_{2}, W$ is always larger than 0.5 .

The polarized direction extraction method reserves two most remarkable direction features for a pixel, one of which is the direction feature of finger vein and the other one is the direction feature of finger dorsal texture. In comparison, conventional methods only reserve the direction feature of finger vein. In this sense, our PWBDC can be more effective. On top of that, the self-adaptive depth-based weighting refines the feature matching according to the gray depth, which can reflect the pixel position information. The position information is discriminative and useful for identification, further improving the effectiveness of the proposed PWBDC method.

\section{Experimental Studies}

\subsection{Experiments on a Newly Established Database}

We first present our experiments and results on a newly established database, which contains 210 independent finger samples. It includes infrared finger patterns viewed from the backside of fingers from both male and female volunteers. The samples in the database were collected in two sessions: the first session for training and the second session for testing. In each session, there are 210 finger samples in the database, and each subject provides index and middle fingers of both left and right hands for capturing. Totally, 55 subjects are recorded in the dataset, with both left and right-hand fingers captured in the same sessions. The time interval between two capture sessions for training and test is 3 months. Samples of index and middle fingers from both sessions are illustrated in Fig. 6, with all the original finger images resized to the same size of $100 \times 240$. In the process of testing, finger samples from the test database will be compared to those in the training database. The class of the sample with the highest matching score in the training database will be selected as the final identified class for the test sample.

Considering that most of previous research on finger vein recognition focuses on the traditional finger vein pattern, as Fig. 2.a shows, we also established another traditional database of this type, which contains the same 210 finger samples, for comparative purposes. Our proposed PWBDC method is tested on both the newly established database of hybrid finger patterns and the traditional database.

Furthermore, we would like to validate the effect on performance improvement induced by the three components of our new hybrid feature, PWBDC: polarized direction extraction, extended normalized angular binary coding, and self-adaptive depth-dependent weighting. To achieve this goal, we design and implement different methods hierarchically integrating different numbers of components.
1. Firstly, to validate the effectiveness of the proposed method, four typical baseline algorithms, LBP [16], LLBP [19], GCC [4], LMC [12], RLT [13] and LDC [33], are also implemented and evaluated on both the traditional database and the newly established database.

2. Secondly, we implement a polarized direction coding (PDC) method, in which the two deepest directions are normally binary coded, and weights of $S_{1}$ and $S_{2}$ are set to be identical, i.e. $W$ is set to 0.5 . In other words, the innovation in the PDC method only includes the introduction of the second deepest direction. Hence we shall use PDC to demonstrate the added value of the second deepest direction, through comparing it with the baselines methods.

3. Thirdly, to validate the effectiveness of the adaptive depthdependent weights, we implement a method of polarized depth-weighted direction coding (PWDC). Compared with PDC, PWDC adds depth-dependent weights; compared with PWBDC, PWDC lacks of the extended binary coding.

4. Finally, we implement PWBDC, which contains all the three components. Hence the different in performance between PWBDC and PWDC can indicate the added value of the extended binary coding.

All methods are executed on the R2014a Matlab platform with 3.30 GHz CPU. As usual, we adopt the recognition accuracy and the equal error rate (EER) as performance measures. In our experiments, the region of interest (ROI) image is extracted, alignment is carried out, and then two measures (recognition accuracy and EER) are calculated using the method in [5].

As Table 2 shows, for all methods, their performance on the newly established database of hybrid finger patterns is better (with higher recognition accuracy and lower EER) than on the traditional database. This indicates that more useful information has been acquired in the hybrid finger pattern that contains both finger vein and finger dorsal texture information. In other words, this verifies the value of the newly established database.

On the other hand, the accuracy of LBP, GCC, LMC, LDC, PDC, PWDC and PWBDC increases (and the EER of them decreases) in oder. From this trend, we can observe the added value of our newly proposed components: 1) The better performance of PDC than LDC indicates the superiority of selecting the two polarized deepest directions; 2) the better performance of PWDC than PDC demonstrates the value of the depth-dependent self-adaptive weights; and 3) the better performance of PWBDC than PWDC implies the added value of the extended binary coding. In summary, the proposed PWBDC method, which combines all three components, demonstrates the highest identification accuracy and the lowest EER, which validates the effectiveness of our proposal.

Moreover, in order to evaluate the performance of different methods further, the false acceptance rate (FAR) and the false rejection rate (FRR) of different methods are calculated, and the DET curves [10] are drawn. As Fig. 7 shows, the DET curve of our proposed PWBDC method is the closest to the bottom-left corner, which indicates that it performs the best compared with all the other compared methods. 


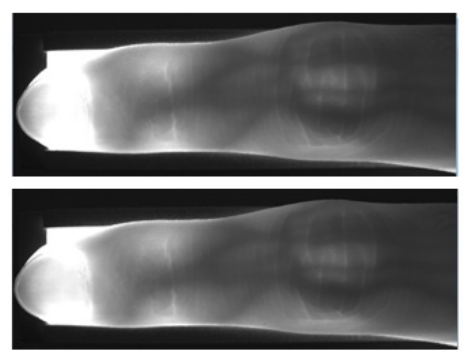

a

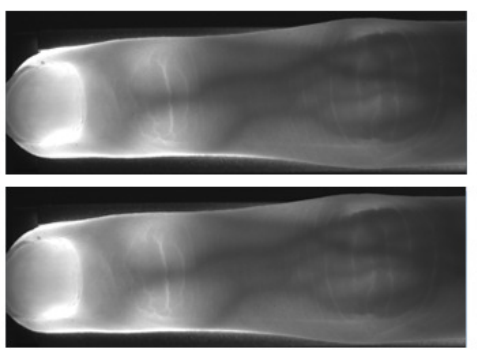

b

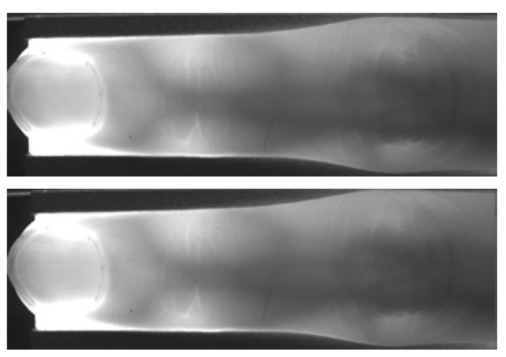

c

Figure 6: Samples of (a) index, (b) middle and (c) ring fingers, respectively. The two images of each column are from the same subject but collected in different sessions.

Table 2: Performance of methods on both the traditional and newly-established databases.

\begin{tabular}{llrrrrrrrrr}
\hline & & LBP & LLBP & GCC & LMC & RLT & LDC & PDC & PWDC & PWBDC \\
\hline Traditional & accuracy (\%) & 86.67 & 88.23 & 87.08 & 89.17 & 88.23 & 89.58 & 91.67 & 92.23 & 93.75 \\
\cline { 2 - 11 } database & EER (\%) & 11.2 & 8.0 & 10.1 & 8.3 & 6.7 & 7.4 & 6.1 & 5.3 & 4.4 \\
\hline $\begin{array}{l}\text { Established } \\
\text { database }\end{array}$ & accuracy (\%) & 90.32 & 91.01 & 91.01 & 92.15 & 92.21 & 94.55 & 95.38 & 96.38 & 98.89 \\
\cline { 2 - 10 } & EER (\%) & 8.3 & 5.3 & 6.7 & 5.7 & 6.4 & 4.5 & 4.1 & 2.5 & 1.3 \\
\hline
\end{tabular}

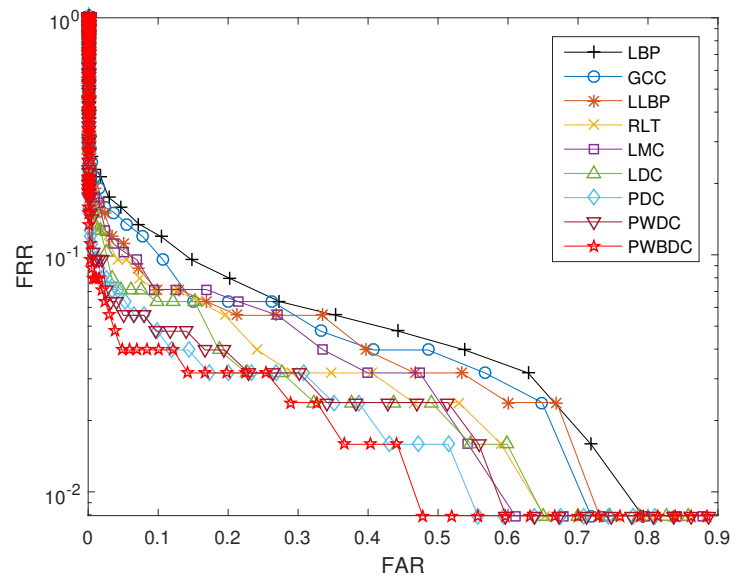

(a) On the traditional database

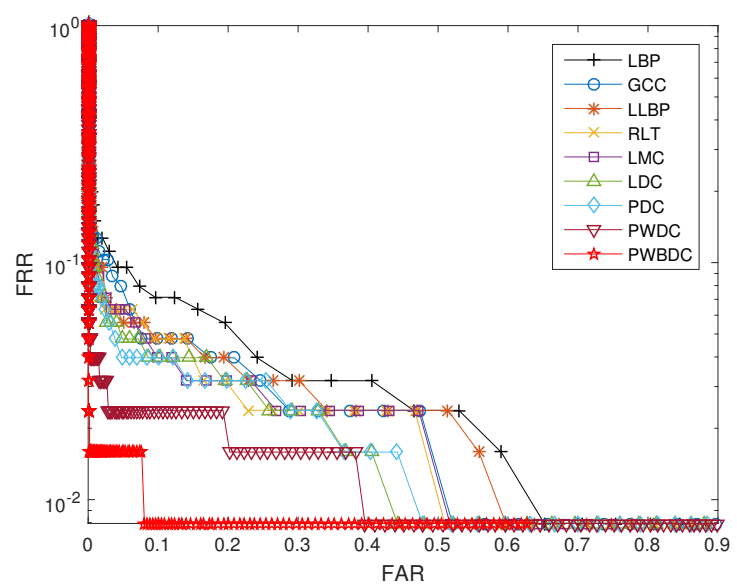

(b) On the newly established database

Figure 7: The DET curves of different methods.
Furthermore, we also test the performances of different algorithms on the index, middle and ring fingers, respectively (Results not shown here). Overall the performance on index fingers is the best and the performance on ring fingers is the worst. This is because the index finger is more flexible and easier to place in a relatively fixed place in the acquisition process, so the process result of which is better than that of the middle and ring fingers.

\subsection{Experiments on a Popular Public Database}

In order to evaluate the proposed PWBDC method more widely, we also conduct experiments on a popular public database HKPU-FV [5]. This database contains 6264 images from 156 volunteers collected in two sessions from April 2009 to March 2010 using a self-designed device, with $93 \%$ of all participants younger than 30 years old. The index finger and the middle finger of each person's left hand are each taken six times in one session.

To compare with the results of different methods with that in [5] and analyze the experiment results more thoroughly, we conduct experiments on the index fingers and the middle fingers separately, as with the experiments in [5]. In each experiment, six finger vein images acquired in the first session are used to build the training set, and the rest six finger vein images acquired in the second session are used to build the test set. All the experiments are done on Matlab 2015b with 4GB RAM.

'Even Gabor with Morphological' (EGM) in Table 3 and Table 4 is the best method for finger vein recognition with both sessions in [5]. As Table 3 and Table 4 show, similar to the case in our established database, the overall identification accuracy of LMC, LDC, PDC, PWDC and PWBDC on the HKPU-FV database increases in order and the overall EER of them decreases in order, which verifies again the effectiveness of PWBDC and its three new components. Moreover, the performance of 
Table 3: Accuracy of methods on different fingers.

\begin{tabular}{lrrr}
\hline accuracy (\%) & Index & Middle & Index\&Middle \\
\hline RLT & 95.1 & 95.0 & 94.2 \\
\hline LMC & 94.4 & 95.9 & 94.8 \\
\hline EGM & 98.1 & 94.1 & 96.1 \\
\hline LDC & 95.9 & 95.9 & 95.5 \\
\hline PDC & 98.4 & 99.1 & 98.7 \\
\hline PWDC & 98.2 & 99.1 & 98.7 \\
\hline PWBDC & 98.6 & 98.9 & 98.7 \\
\hline
\end{tabular}

Table 4: EER of methods on different fingers.

\begin{tabular}{lrrr}
\hline EER $(\%)$ & Index & Middle & Index\&Middle \\
\hline RLT & 7.3 & 7.8 & 7.4 \\
\hline LMC & 5.6 & 5.2 & 5.1 \\
\hline EGM & 3.3 & 7.0 & 4.9 \\
\hline LDC & 4.9 & 4.6 & 4.6 \\
\hline DeepVein & 1.9 & 4.1 & 3.1 \\
\hline CNN+FCN & 1.7 & 3.5 & 2.7 \\
\hline PDC & 2.6 & 2.2 & 2.4 \\
\hline PWDC & 2.5 & 2.3 & 2.4 \\
\hline PWBDC & 2.4 & 2.3 & 2.2 \\
\hline
\end{tabular}

our proposed PWBDC method is better than that of EGM. Besides these conventional methods, two other deep learning-based methods, namely DeepVein [3] and CNN+FCN [18], are carried out on the HKPU-FV database in our experiments and the results of EER are shown in Table 4. Compared with these two deep learning-based methods, our proposed approach achieves lower overall EER indicating better performance.

Fig. 8 shows the DET curves of different methods on this database. The HKPU-FV database is a public database with traditionally captured finger veins. However, even in this case, the proposed PWBDC method still performs excellently on it, which may indicate PWBDC's stronger ability of feature extraction and better strategies in score calculation.

Nevertheless, despite the extraordinary performance of the proposed PWBDC method on our newly established database and the public HKPU-FV database, we have found that failed identification cases may happen under extreme conditions such as very distinct light variation and large finger rotation. To tackle such cases is one of our future work.

\section{Conclusions and Future Work}

In this paper, for finger-based biometric identification, we have proposed a new system to acquire dorsal finger vein and texture in a single image, and we have proposed a new hybrid feature and its extraction method called PWBDC. We have showed that the proposed system and feature are not only storagesaving, but more importantly identification-effective. We have also established a new database of such hybrid images of 210 finger samples. Experimental results have demonstrated that

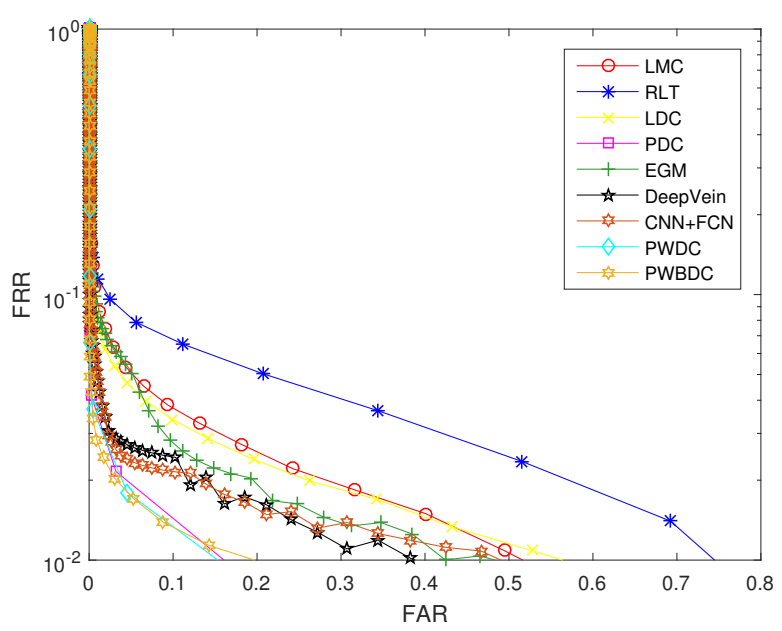

Figure 8: The DET curves of algorithms on the HKPU-FV database.

the proposed PWBDC method performed superiorly on both the newly established database of hybrid images and a popular public database of traditional finger vein images, better than many established and state-of-the-art methods.

Our future work includes: firstly, we will expand further our established database; secondly, we shall tackle the failure cases of PWBDC, starting from developing an enhanced preprocessing method; thirdly, some other modalities could also be added into the identification process, such as fingerprint or finger knuckle texture, to make an even more reliable and robust finger identification system; finally, a novel feature extraction method, called multi-orientation weighted symmetric local graph structure for finger veins, was proposed in [1], which currently cannot be applied to our data because of the added finger dorsal textures; it would be interesting to investigate the extension of this method to our hybrid finger pattern.

\section{Acknowledgment}

This work was partly supported by the National Natural Science Foundation of China (No.61471216 and No.61771276), and the Special Foundation for the Development of Strategic Emerging Industries of Shenzhen (No.JCYJ20170817161845824, No.JCYJ20170307153940960 and No.JCYJ20150831192224146).

\section{References}

[1] Dong, S., Yang, J., Chen, Y., Wang, C., Zhang, X., Park, D.S.: Finger vein recognition based on multi-orientation weighted symmetric local graph structure. KSII Transactions on Internet \& Information Systems 9(10) (2015)

[2] Hashimoto, J.: Finger vein authentication technology and its future. In: VLSI Circuits, 2006. Digest of Technical Papers. 2006 Symposium on, pp. 5-8. IEEE (2006)

[3] Hong, H.G., Lee, M.B., Park, K.R.: Convolutional neural network-based finger-vein recognition using NIR image sensors. Sensors 17(6), 1297 (2017)

[4] Kong, A.K., Zhang, D.: Competitive coding scheme for palmprint verification. In: Pattern Recognition, 2004. ICPR 2004. Proceedings of the 17th International Conference on, vol. 1, pp. 520-523. IEEE (2004) 
[5] Kumar, A., Zhou, Y.: Human identification using finger images. IEEE Transactions on Image Processing 21(4), 2228-2244 (2012)

[6] Kumar, A., Zhou, Y.: Human identification using finger images. IEEE Transactions on image processing 21(4), 2228-2244 (2012)

[7] Lee, E.C., Jung, H., Kim, D.: New finger biometric method using near infrared imaging. Sensors 11(3), 2319-2333 (2011)

[8] Lee, T.S.: Image representation using $2 \mathrm{~d}$ gabor wavelets. IEEE Transactions on Pattern Analysis and Machine Intelligence 18(10), 959-971 (1996)

[9] Lee, Y., Khalil-Hani, M., Bakhteri, R.: FPGA-based finger vein biometric system with adaptive illumination for better image acquisition. In: Computer Applications and Industrial Electronics (ISCAIE), 2012 IEEE Symposium on, pp. 107-112. IEEE (2012)

[10] Martin, A., Doddington, G., Kamm, T., Ordowski, M., Przybocki, M.: The DET curve in assessment of detection task performance. Tech. rep., DTIC Document (1997)

[11] Miura, N., Nagasaka, A., Miyatake, T.: Feature extraction of finger-vein patterns based on repeated line tracking and its application to personal identification. Machine Vision and Applications 15(4), 194-203 (2004)

[12] Miura, N., Nagasaka, A., Miyatake, T.: Extraction of finger-vein patterns using maximum curvature points in image profiles. IEICE TRANSACTIONS on Information and Systems 90(8), 1185-1194 (2007)

[13] Miura N Nagasaka A, M.T.: Feature extraction of finger-vein patterns based on repeated line tracking and its application to personal identification. Machine Vision and Applications (2004)

[14] Mobarakeh, A.K., Rizi, S.M., Khaniabadi, S.M., Bagheri, M.A., Nazari, S.: Applying weighted k-nearest centroid neighbor as classifier to improve the finger vein recognition performance. In: Control System, Computing and Engineering (ICCSCE), 2012 IEEE International Conference on, pp. 56-59. IEEE (2012)

[15] Mulyono, D., Jinn, H.S.: A study of finger vein biometric for personal identification. In: Biometrics and Security Technologies, 2008. ISBAST 2008. International Symposium on, pp. 1-8. IEEE (2008)

[16] Ojala, T., Pietikaeinen, M., Maeenpaeae, T.: Multiresolution gray-scale and rotation invariant texture classification with local binary patterns. IEEE Transactions on Pattern Analysis and Machine Intelligence 24(7), 971-987 (2002)

[17] Qin, H., El-Yacoubi, M.A.: Deep representation based feature extraction and recovering for finger-vein verification. IEEE Transactions on Information Forensics and Security (2017)

[18] Qin, H., El-Yacoubi, M.A.: Deep representation-based feature extraction and recovering for finger-vein verification. IEEE Transactions on Information Forensics and Security 12(8), 1816-1829 (2017)

[19] Rosdi, B.A., Shing, C.W., Suandi, S.A.: Finger vein recognition using local line binary pattern. Sensors 11(12), 11,357-11,371 (2011)

[20] Varun, C., Lalith, K.B.S., Bhatnagar, S., et al.: Finger vein authentication using local ternary co-occurrence pattern feature descriptor. In: Inventive Computation Technologies (ICICT), International Conference on, vol. 3 , pp. 1-6. IEEE (2016)

[21] Vega, A.P., Travieso, C.M., Alonso, J.B.: Biometric personal identification system based on patterns created by finger veins. In: Bio-inspired Intelligence (IWOBI), 2014 International Work Conference on, pp. 6570. IEEE (2014)

[22] Veluchamy, S., Karlmarx, L.: A system for multimodal biometric recognition based on finger knuckle and finger vein using feature level fusion and k-svm classifier. IET Biometrics (2016)

[23] Vlachos, M., Dermatas, E.: Finger vein segmentation from infrared images based on a modified separable Mumford Shah model and local entropy thresholding. Computational and mathematical methods in medicine 2015 (2015)

[24] Wu, J.D., Liu, C.T.: Finger-vein pattern identification using principal component analysis and the neural network technique. Expert Systems with Applications 38(5), 5423-5427 (2011)

[25] Wu, J.D., Liu, C.T.: Finger-vein pattern identification using SVM and neural network technique. Expert Systems with Applications 38(11), 14,284-14,289 (2011)

[26] Xu, J., Jianjiang, C., Dingyu, X., Feng, P.: Near infrared vein image acquisition system based on image quality assessment. In: Electronics, Communications and Control (ICECC), 2011 International Conference on, pp. 922-925. IEEE (2011)

[27] Yang, J., Shi, Y.: Finger-vein ROI localization and vein ridge enhance- ment. Pattern Recognition Letters 33(12), 1569-1579 (2012)

[28] Yang, J., Shi, Y.: Towards finger-vein image restoration and enhancement for finger-vein recognition. Information Sciences 268, 33-52 (2014)

[29] Yang, J., Zhang, X.: Feature-level fusion of fingerprint and finger-vein for personal identification. Pattern Recognition Letters 33(5), 623-628 (2012)

[30] Yang, L., Yang, G., Yin, Y., Xi, X.: Exploring soft biometric trait with finger vein recognition. Neurocomputing 135, 218-228 (2014)

[31] Yang, W., Huang, X., Zhou, F., Liao, Q.: Comparative competitive coding for personal identification by using finger vein and finger dorsal texture fusion. Information Sciences 268, 20-32 (2014)

[32] Yang, W., Ji, W., Liao, Q.: Significance of being unique from finger patterns: Exploring hybrid near-infrared finger vein and finger dorsal patterns in verifying human identities. In: Chinese Conference on Biometric Recognition, pp. 529-535. Springer (2015)

[33] Yang, W., Rao, Q., Liao, Q.: Personal identification for single sample using finger vein location and direction coding. In: Hand-Based Biometrics (ICHB), 2011 International Conference on, pp. 1-6. IEEE (2011) 